\title{
Coordination Control of FSCWT for Enhancing LVRT Capability Using Hierarchical Multi-object Predictive Strategy
}

\author{
Hongwei Li* and Haiying Dong \\ School of Automation and Electrical Engineering, Lanzhou Jiaotong University \\ lihongwei@mail.lzjtu.cn*,hydong@mail.lzjtu.cn
}

\begin{abstract}
This paper presents a coordinated approach for enhancing the low voltage ride through capability of the front-end speed controlled wind turbine (FSCWT) with a hierarchical multi-object predictive strategy applied. The hierarchical multi-object predictive strategy has the ability not only to handle the nonlinearities existed in the pitch control, excitation control and speed regulation processes, but also can avoid the shortcomings of weighted coefficient method and lexicographic method during the process of multi-objective dynamic optimization. The proposed method is realized using the improved genetic algorithm. Simulations are conducted to verify the effectiveness of the coordination control scheme with the proposed method. Simulation results show that the proposed method can enhance the LVRT capability greatly when compare with conventional voltage control approach, the output power and the rotor speed are also optimized during the coordination process.
\end{abstract}

Keywords: Coordination; low voltage ride though; multi-object predictive strategy; front-end speed control wind turbine

\section{Introduction}

Facing with the ever-increasing amount of grid-connected wind turbines and publication of different grid codes for wind turbines (WTs), it is essential to promote control technologies of WT to meet such requirements. Among all, the low voltage ride through (LVRT) capability is of the greatest importance that affects the stability and safety of WT or/and even the entire system.

As the majority of grid-connected wind turbines are doubly-fed induction generators (DFIGs) with converters used, high penetration levels of wind power have result in great influences on the operation of the grid it integrated [1,2]. To fulfill the LVRT requirements, rotor side of DFIG would overcurrent in the case when the grid voltage sagged [3], the rapid increasing of current in rotor side, at the same time, would lead to DC side voltage of the excitation converter soar and finally causes an oscillation of the current, and both the active and reactive power of the converter [4, 5]. Current strategies for coping with LVRT problems are mainly focus on rotor side protection with crowbar, new converter topology developing and advanced excitation algorithms applying. However, to help the WTs with DFIGs to ride through a low voltage once these measures applied, the total costs as well as the control complexity would be increased in certain extent $[6,7]$. Furthermore, regular short-circuit protection would result in a flowing of reactive power from grid to WT side during voltage sagging [8].

Compared with WTs with DFIGs, the front-end speed controlled wind turbines (FSCWT) proposed in this paper, employs a hydro-dynamic controlled gearbox instead of the electrical inverter and directly connected to grid by an electrically excited synchronous generator (EESG) with rated voltage up to $10 \mathrm{kV}$ without any transformer used, which was presented in Figure 1 [9]. Due to the adoption of new drivetrain, the main aim of the control system is to adjust the speed and torque of the torque converter 
WinDrive, therefore to cooperate with the pitch controller and the excitation controller and finally guarantee the turbine works with maximum efficiency and high power quality, especially the LVRT capability during severe faults.

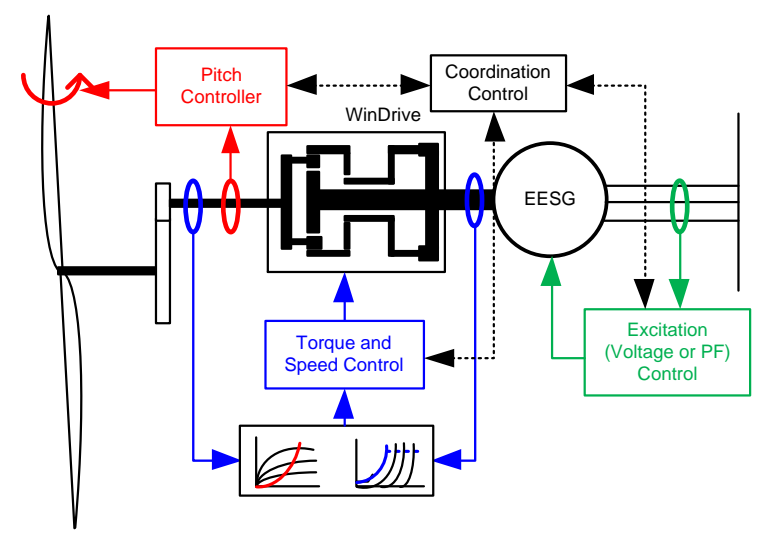

Figure 1. Front-end Speed Controlled Wind Turbine

In this paper, a coordinated strategy for obtain technically justified LVRT requirements of FSCWT based on system characteristics is proposed. The objective is to ensure an efficient operation during normal operation and meet the LVRT requirements for WTs. Thus, helps to obtain a reliable and safe operation once grid faults happened. The rest of this paper is organized as follows. Section II introduces the operation principle of FSCWT in different operation zone under Chinese grid connection requirements. In Section III, a hierarchical strategy for LVRT control under different operation conditions is presented. Section IV implements how the proposed strategy works for obtaining a coordination of the variable pitch as well as the torque control of FSCWT. Simulations on a local network technically justified LVRT requirements for FSCWT are conducted, the results obtained by the application of the proposed approach are presented in Section V. Finally, the conclusions are given in Section VI.

\section{Operation Principle of FSCWT}

Generally, the LVRT of WT that installed with DFIG is realized by pitch control, converter control and power factor (PF) control. Among all, the LVRT control strategy for FSCWT is the most complex, which needs the pitch control, the guide vane control (namely, torque and speed control of WinDrive ), and the excitation control to cooperate with each other. In the first stage (shown as the operation zone I in Figure 2), the rotation speed of wind rotor stays at a relatively low value while the wind speed just below a cutin speed. In this stage, the guide vane control plays as a main role in accelerating the main shaft speed. In the second stage, as the main shaft speed reaches near a synchro-speed, it would be the time for grid connection. At the same time, the excitation controller would be activated. After WT connected to the grid, the turbine would work in a power optimal stage, namely in the operation zone III. During this period, the pitch controller and guide vane controller are coordinated to achieve an optimal control of captured power. When the rotor speed $\omega$ reaches near rated level $\omega_{\mathrm{R}}$, the WT would work in a speed control mode in zone IV until the output power grows near a rated level $P_{\mathrm{R}}$. After that, the turbine would operate in zone $\mathrm{V}$ for power control.

In reality, the WT usually works in operation zone II to $\mathrm{V}$ as wind speed always varies from a cut-in speed to a rated level. As is shown in Figure 2, the pitch controller, the guide vane controller and the excitation controller almost work together all the time. To obtain maximum power under synchro-speed and meet LVRT requirements under special circumstances, it is needed for the coordinated controller to balance between pitch control, 
torque control and excitation control for realizing such objective.

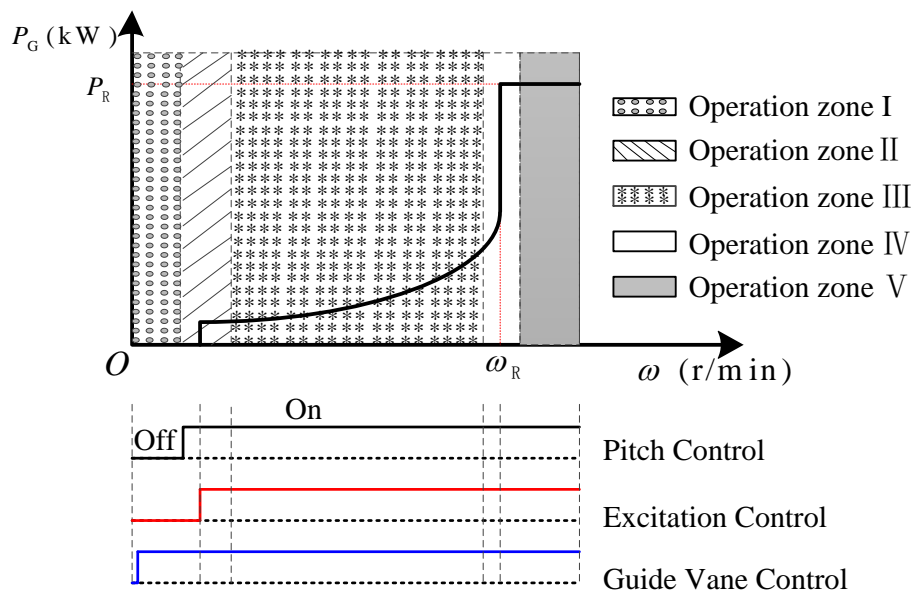

Figure 2. Speed-Power Curve and Operation Zone of FSCWT

During operation process, the pitch controller acts as an auxiliary unit of WT for realizing LVRT in high wind speed. Meanwhile, the guide vane controller functions as a damper to absorb or release part of the energy released by WinDrive during wind speed variation or severe faults happened in the grid-side. Thus, the imbalance between electromagnetic power of gird-side and mechanical power from wind rotor can be reduced. At the same time, the excitation controller regulates the excitation current according to the variation of the terminal voltage of the EESG and keeps it to a set value, and then guarantees a dynamic and transient stability of the whole generation system in LVRT process.

During voltage sagging, all the three subsystems, namely the pitch control system, the guide vane control system and the excitation system, can reduce the power imbalance of electromagnetic power and mechanical power. The pitch control and guide vane control mainly works to decrease the input mechanical power of the prime turbine while the excitation control functions to increase the electromagnetic power. Therefore, it is the combination of the three systems that make it possibly for FSCWT to realize LVRT.

\section{LVRT Control Strategy for FSCWT}

Generally, the safe operation and the condition monitoring would be done by the main controller, for FSCWT, which needed coordination of the pitch control, the guide vane control and the excitation control under different wind speed, terminal voltage or operation state of the turbine. Thus, coordination control was needed especially in the LVRT control process.

\subsection{LVRT Requirements for Wind Turbines}

To ensure a safe and stable operation of the WTs, various grid codes have been drawn up by power utilities as well as power administrative department [10-13], which consist of requirements for WTs or/and wind farms. The requirements for LVRT performance of E.ON in Germany and Sate Grid of China were presented in Figure 3. 


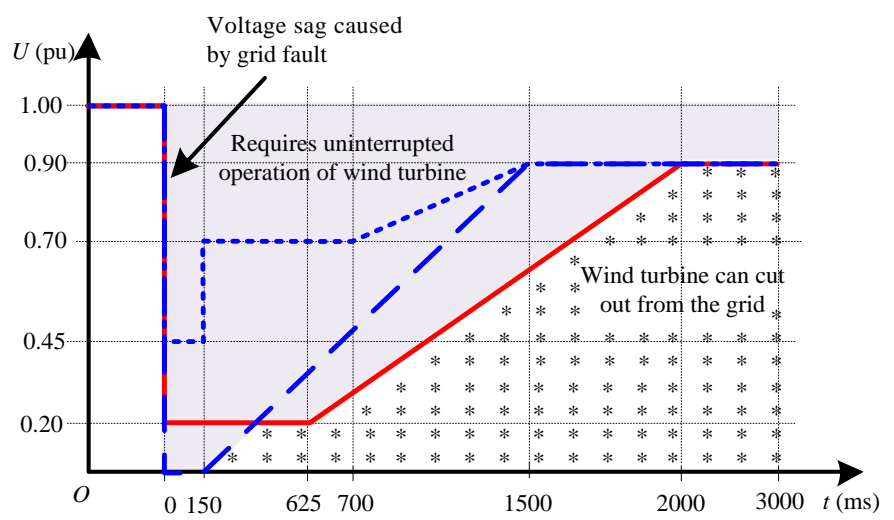

Figure 3. Requirements on LVRT for Wind Turbine of Different Grid Codes

The State Grid codes defined that every WT connected to power grid must fulfill the LVRT requirements donated as red solid line in Figure 3. When the voltage of the gridside fallen to $20 \%$ of the rated voltage as transient faults happened in grid-side, the WTs must keep in a grid-connection state for at least $625 \mathrm{~ms}$ and generate reactive power to the grid-side to assist the voltage recovery. Once the fault was cleared, the point of common coupling (PCC) voltage should return to $90 \%$ of the rated value in $2 \mathrm{~s}$. In contrast, the grid code of E.ON power utility was of higher requirements, which cleared the WT must remain connected to power grid for $150 \mathrm{~ms}$ during severe fault happen in grid-side even the PCC voltage fallen to zero. Once the fault was cleared, the PCC voltage should get back to $90 \%$ of the rated value in $1.5 \mathrm{~s}$.

\subsection{Structure of the Coordination Controller}

In order to guarantee the FSCWT meet the LVRT requirements, all subcomponent should work together and cooperated with each other. Moreover, a LVRT controller was needed to coordinate with the pitch controller, the guide vane controller and excitation controller. Thus, a control scheme for LVRT control of FSCWT was proposed, which was shown in Figure 4.

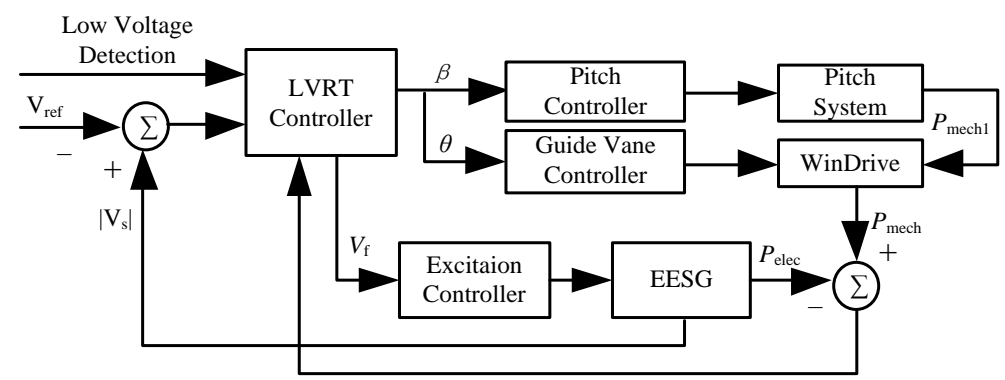

Figure 4. Scheme of the Coordination Controller

During operation process, the PCC voltage was continuously detected by the safety control system of the WT, and the detected signal was sent to LVRT controller online. If the detected PCC voltage stays in a normal level, the pitch controller and guide vane controller would operate in a normal state, which means the pitch angle and the guide vane angle was given mainly depend on wind speed. Figure 5 and Figure 6 gives the pitch angle and the guide vane angle calculation diagram. Considering the limitation of the context, the procedures for pitch angle calculation would not be introduced in details. In the first step for guide vane angle calculation, an operation zone would be selected 
according to the wind speed difference $\Delta v$ calculated from comparison with the measured real time wind speed and the rated one, which consisting two situations:

1) If $\Delta v<0$, namely the real time wind speed is less than the rated one, the WT would work in operation zone IV as shown in Figure 2 while the switch needed turn to the ' 2 ' in Figure 6 . The given speed of the rotor $\omega_{R \mathrm{~s}}$ should be calculated by wind speed $v$ and its corresponding best tip speed ratio, which was compared with the measured rotor speed $\omega_{\mathrm{R}}$ and result in $\Delta \omega_{R}$ as an input for guide vane controller.

2) If $\Delta v \geq 0$, the switch stays at ' 1 ' while the WT works in operation zone III. Then the rated wind speed was utilized as input variable for speed control of WT. However, no matter the coordination controller works in operation zone III or IV, the EESG speed $\omega_{\mathrm{G}}$ and the pump wheel speed $\omega_{\mathrm{P}}$ of WinDrive keep in a synchronous speed: $\omega_{\mathrm{G}}=\omega_{\mathrm{P}}=1500$ $\mathrm{r} / \mathrm{min}$. Meanwhile, the comparison result $\Delta \omega_{\mathrm{G}}$ of EESG speed $\omega_{\mathrm{G}}$ and the set value $\omega_{\mathrm{R}}$ was used as another input of the guide vane controller (see in Figure 6).

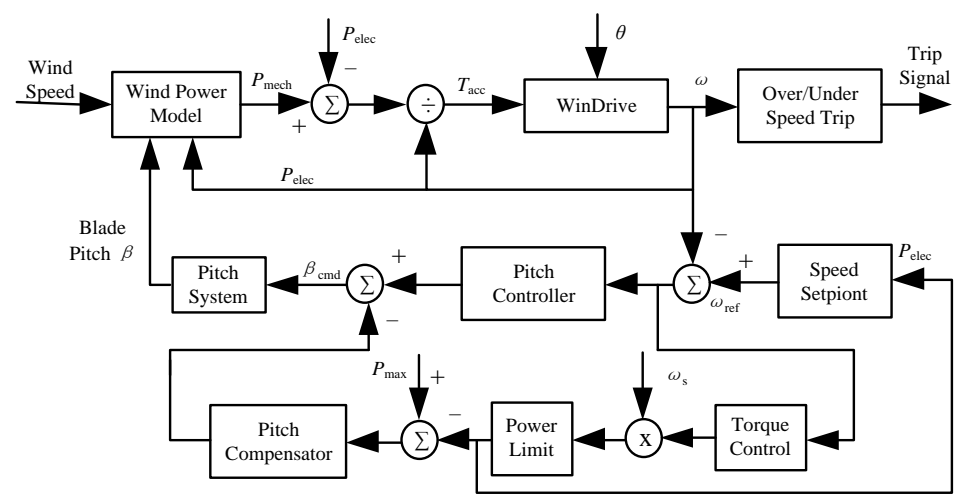

Figure 5. Pitch Angle Calculation Diagram

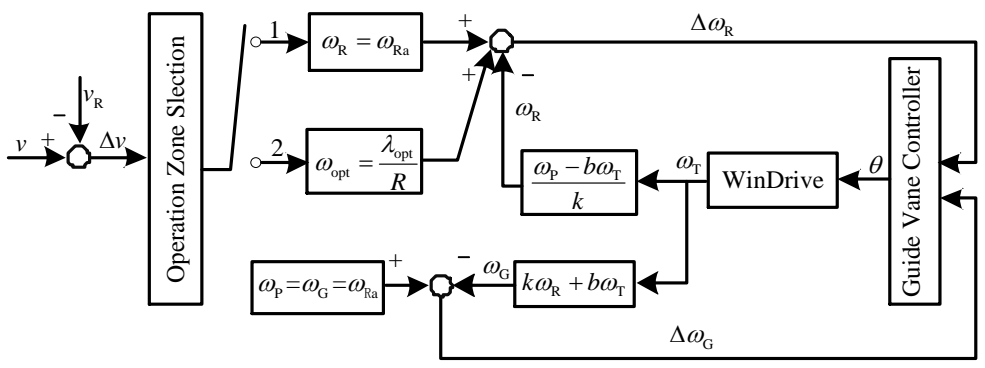

Figure 6. Guide Vane Angle Calculation Diagram

However, once a low voltage signal was detected from PCC, the LVRT controller would start to work. The pitch angle $\beta$ and guide vane angle $\theta$ no longer given by wind speed variation, but decided by voltage detected from PCC as well as the difference of mechanical power and the electromagnetic power. This relationship was shown in Figure 4. Moreover, the excitation voltage would also be regulated via LVRT controller, which was in accordance with the control structure shown in Figure 1.

\section{Hierarchical Multi-object Predictive Strategy}

\subsection{Hierarchical Multi-objective Genetic Algorithm}

Without loss of generality, assuming the multi-objective optimization problem $g=\left\{g_{1}\right.$, $\left.g_{2}, \cdots, g_{n}\right\}$ contains $n$ objectives, and every objective $g_{j}(j=1,2, \cdots, n)$ needed to be 
minimized. These objectives were sorted according to its relative importance in $l(1 \leq l)$ priorities. Thus, the optimization problem can be re-expressed as: $G=\left\{G_{1}, G_{2}, \cdots, G_{n}\right\}$, where, $G_{i}=\sum_{j} \lambda_{i j} g_{i j}$ stands for the composite objective function of $j$ th objective with $i$ th priority. The subscript of the objective $G_{i}$ also represents the relative importance of the $i$ th priority. According to [14], we can construct hierarchical multi-objective genetic algorithm. Assuming for every composite objective function of $G=\left\{G_{1}, G_{2}, \cdots, G_{n}\right\}$, there exists a corresponding fitness function $F_{i} \in[0,1](1 \leq i \leq l)$, and $F_{i}=1$ represents the objective is completely satisfied. Introducing a set of dynamic coefficient

$$
\delta_{i}= \begin{cases}1 & i=1 \\ 1 & F_{1}=F_{2}=\cdots=F_{i-1}=1,2 \leq i \leq 1 \\ 0 & \text { otherwise }\end{cases}
$$

Thus, the fitness function of the multi-objective optimization problem can be donated as

$$
F=\delta_{1} F_{1}+\delta_{2} F_{2}+\cdots+\delta_{l} F_{l}=\sum_{i=1}^{l} \delta_{i} F_{i}
$$

In Equation (2), as the objective with the first priority is of the most importance, its corresponding fitness functions $F_{i}$ needs to be taken into consideration all the time, and fulfills $\delta_{i}=1$ in Equation (1). Other fitness functions of the objectives with $i^{\text {th }}$ priority would be considered, and at this time $\delta_{i}=1$, only if the objectives are of higher priorities.

As the fitness function is the only window for drawing the external information into genetic algorithm in optimization process, corresponding hierarchical multi objective genetic algorithm can be obtained by applying the fitness function of Equation (2). Whereas, the encoding approach and the genetic operators which including crossover, mutation, and selection can be selected automatically on the basis of actual situation of the optimization problem.

\subsection{Multi-Objective Predictive Controller Based On Hierarchical Genetic Algorithm}

The multi-objective nonlinear controller corresponding to the above-mentioned genetic algorithm [15] can be directly constructed, the solving steps of the control variables are:

1) Generating $M$ solutions randomly within the scope of control variable constraint;

2) Generating $M$ progenies using crossover and mutation, then selecting $M$ solutions with higher fitness from the $2 M$ solution constituted of both parent solutions and progeny ones as the new parent population. The evolution process will repeat until the conditions for the termination of the evolutionary computation is fulfilled;

3) Exporting the current solutions with the highest fitness after termination of evolutionary computation, and donate the instant control variable of the current controller as the corresponding control variable.

\subsection{Model and Control Objectives of LVRT Controller}

\subsubsection{System Modeling}

In order to analyze the dynamic characteristics of transmission system shown in Figure 1 simplistic, the driven train is equivalent to a multi-mass-block model [16, 17]. The driven train presented in Figure 1 can be expressed as:

$$
J_{\mathrm{R}} \dot{\omega}_{\mathrm{R}}=T_{\mathrm{R}}-T_{\mathrm{j}}-D \omega_{\mathrm{R}}
$$

where $J_{\mathrm{R}}$ and $\omega_{\mathrm{R}}$ represent the inertia and rotation speed of the rotor, respectively. $D$ is the damping coefficient of the low speed shaft, and $T_{\mathrm{R}}$ is the torque of wind wheel.

$$
T_{\mathrm{R}}=P_{1} / \omega_{\mathrm{R}}=C_{\mathrm{p}}(\lambda, \beta) A_{\mathrm{r}} v^{3} \rho / 2 \omega_{\mathrm{R}}
$$

For the hydro-dynamic gearbox WinDrive, the dynamic equilibrium Equation [18] of pump wheel and turbine shaft can be described as: 


$$
\left\{\begin{array}{l}
J_{\mathrm{t}} \dot{\omega}_{\mathrm{P}}=T_{\mathrm{t}}-T_{\mathrm{P}}-T_{\mathrm{G}} \\
J_{\mathrm{q}} \dot{\omega}_{\mathrm{T}}=T_{\mathrm{q}}-T_{\mathrm{T}}
\end{array}\right.
$$

where $J_{\mathrm{t}}$ and $J_{\mathrm{q}}$ stand for rotation inertia of the main shaft and the outer ring gear, respectively. $T_{\mathrm{t}}, T_{\mathrm{p}}, T_{\mathrm{q}}, T_{\mathrm{T}}$, and $T_{\mathrm{G}}$ present the mechanic torque of the main shaft, pump wheel, ring gear, turbine wheel and the EESG, respectively. According to the speed relationships given in [18],

$$
\dot{\omega}_{\mathrm{T}}=\frac{1+i_{\mathrm{Tq}}}{1+i_{\mathrm{tq}}}\left(\dot{\omega}_{\mathrm{B}}-i_{\mathrm{Rj}} i_{\mathrm{tq}} \dot{\omega}_{\mathrm{R}}\right)
$$

Without taking the rotation inertia of high speed shaft into consideration, the dynamical characteristics of the high speed shaft and the EESG can be described as:

$$
J_{\mathrm{G}} \dot{\omega}_{\mathrm{G}}=T_{\mathrm{e}}-T_{\mathrm{G}}-D_{\mathrm{G}} \omega_{\mathrm{G}}
$$

where $i_{\mathrm{Tq}}$ is the speed ratios of turbine wheel and ring gear, and $i_{\mathrm{tq}}$ is the ratio of the main shaft and ring gear, $i_{\mathrm{Rj}}$ is the ratio of the rotor and carrier. $J_{\mathrm{G}}$ is the rotor inertia of EESG and $D_{\mathrm{G}}$ is the corresponding damping coefficient.

Consider a general plant of the synchronous generator in [19], as represented in Park's framework:

$$
\left\{\begin{array}{l}
v_{\mathrm{d}}=-R_{\mathrm{s}} i_{\mathrm{d}}-p \psi_{\mathrm{d}}+\omega_{\mathrm{e}} \psi_{\mathrm{q}} \\
v_{\mathrm{q}}=-R_{\mathrm{s}} i_{\mathrm{q}}+p \psi_{\mathrm{q}}+\omega_{\mathrm{e}} \psi_{\mathrm{d}} \\
v_{\mathrm{f}}=p \psi_{\mathrm{f}}+R_{\mathrm{f}} i_{\mathrm{f}} \\
0=p \psi_{\mathrm{D}}+R_{\mathrm{D}} i_{\mathrm{D}} \\
0=p \psi_{\mathrm{Q}}+R_{\mathrm{Q}} i_{\mathrm{Q}}
\end{array}\right.
$$

where $i_{\mathrm{d}}$ and $i_{\mathrm{q}}$ are respectively the direct and transverse currents, $i_{\mathrm{D}}$ and $i_{\mathrm{Q}}$ are the direct and transverse damper's currents and $i_{\mathrm{f}}$ is the exciter current. $\psi_{\mathrm{d}}$ and $\psi_{\mathrm{q}}$ are the stator total flux, $\psi_{\mathrm{f}}$ is the main field total flux. $\psi_{\mathrm{D}}$ and $\psi_{\mathrm{Q}}$ are the direct and transverse damper's total flux. $R_{\mathrm{s}}, R_{\mathrm{f}}$ are respectively the stator resistance and the main field resistance, $R_{\mathrm{D}}$ and $R_{\mathrm{Q}}$ are the damper's resistances. $\omega_{\mathrm{e}}$ is the electrical speed corresponding to the time derivative of the stator electrical angle.

The power Equation of synchronous generator can be written as:

$$
P_{\mathrm{e}}=\left[E_{\mathrm{q}}^{\prime}+\left(X_{\mathrm{q}}-X_{\mathrm{d}}^{\prime}\right) i_{\mathrm{d}}\right] i_{\mathrm{q}}-R_{\mathrm{s}}\left(i_{\mathrm{d}}^{2}+i_{\mathrm{q}}^{2}\right)
$$

where, $P_{\mathrm{e}}$ is the electromagnetic power of EESG, $E_{q}^{\prime}$ is the transverse electromotive force, $X_{\mathrm{d}}$ and $X_{\mathrm{d}}^{\prime}$ are respectively the direct resistances and transient resistance, respectively.

By ignoring the damper winding response, the transient response of stator winding and the effect on rotor speed variation, a simplified synchronous generator in Park's framework can be defined as:

$$
\left\{\begin{array}{l}
T_{\mathrm{d} 0}^{\prime} \dot{E}_{\mathrm{q}}^{\prime}=-E_{\mathrm{f}}-E_{\mathrm{q}}^{\prime}-\left(X_{\mathrm{d}}-X_{\mathrm{d}}^{\prime}\right) i_{\mathrm{d}} \\
v_{\mathrm{d}}=-R_{\mathrm{s}} i_{\mathrm{d}}+X_{\mathrm{q}} i_{\mathrm{q}} \\
v_{\mathrm{q}}=-R_{\mathrm{s}} i_{\mathrm{q}}-X_{\mathrm{d}}^{\prime} i_{\mathrm{d}}+E^{\prime}
\end{array}\right.
$$

where, $X_{\mathrm{q}}$ is the transverse resistances of EESG, and $E^{\prime}$ is the transient potential of EESG. $E_{\mathrm{f}}$ is the field potential. $T_{\mathrm{d} 0}^{\prime}$ is the transient time constant of the d-axis.

Combined with (8) and (9), we can get a three order order excitation control model of synchronous generator: 


$$
\left\{\begin{array}{l}
\frac{\mathrm{d} E_{\mathrm{q}}^{\prime}}{\mathrm{d} t}=\frac{1}{T_{\mathrm{d} 0}^{\prime}} E_{\mathrm{f}}-\frac{1}{T_{\mathrm{d} 0}^{\prime}} E_{\mathrm{q}}^{\prime}-\frac{\left(X_{\mathrm{d}}-X_{\mathrm{d}}^{\prime}\right)}{T_{\mathrm{d} 0}} I_{\mathrm{d}} \\
\frac{\mathrm{d} E_{\mathrm{q}}^{\prime \prime}}{\mathrm{d} t}=\frac{c}{T_{\mathrm{d} 0}^{\prime}} E_{\mathrm{f}}+\left(\frac{1}{T_{\mathrm{d} 0}^{\prime \prime}}-\frac{c}{T_{\mathrm{d} 0}^{\prime}}\right) E_{\mathrm{q}}^{\prime}-\frac{E_{\mathrm{q}}^{\prime \prime}}{T_{\mathrm{d} 0}^{\prime \prime}}-\left(\frac{X_{\mathrm{d}}^{\prime}-X_{\mathrm{d}}^{\prime \prime}}{T_{\mathrm{d} 0}^{\prime \prime}}+\frac{c X_{\mathrm{d}}^{\prime}-c X_{\mathrm{d}}^{\prime \prime}}{T_{\mathrm{d} 0}^{\prime}}\right) I_{\mathrm{d}} \\
\frac{\mathrm{d} E_{\mathrm{d}}^{\prime \prime}}{\mathrm{d} t}=-\frac{1}{T_{\mathrm{q} 0}^{\prime \prime}} E_{\mathrm{d}}^{\prime \prime}+\frac{X_{\mathrm{q}}-X_{\mathrm{d}}^{\prime \prime}}{T_{\mathrm{d} 0}^{\prime \prime}} I_{\mathrm{d}} \\
\dot{E}_{\mathrm{f}}=-\frac{E_{\mathrm{f}}}{\tau_{\mathrm{m}}+3 T_{\mathrm{s}}}+\frac{K_{\mathrm{PWM}} X_{\mathrm{ad}}}{\left(\tau_{\mathrm{m}}+3 T_{\mathrm{s}}\right) R_{\mathrm{f}}} U_{\mathrm{C}}
\end{array}\right.
$$

where $U_{\mathrm{C}}$ is the output voltage of excitation controller, $\mathrm{c}=\left(X_{\mathrm{d}}^{\prime \prime}-X_{1}\right) /\left(X_{\mathrm{d}}^{\prime}-X_{1}\right)$.

In (10), let $y=E_{q}^{\prime}$, the Equation of the electrically excited synchronous containing the excitation system can be written as:

$$
\left\{\begin{array}{l}
\dot{\boldsymbol{X}}=\boldsymbol{A}_{\mathrm{P}} \boldsymbol{E}+\boldsymbol{B}_{\mathrm{P} 1} \boldsymbol{I}+\boldsymbol{B}_{\mathrm{P} 2} U_{\mathrm{C}} \\
\boldsymbol{Y}=\boldsymbol{C}_{\mathrm{P}} \boldsymbol{E}
\end{array}\right.
$$

with

$$
\begin{aligned}
& \boldsymbol{X}=\left[\begin{array}{c}
E_{\mathrm{f}} \\
E_{\mathrm{q}}^{\prime} \\
E_{\mathrm{q}}^{\prime \prime} \\
E_{\mathrm{d}}^{\prime \prime}
\end{array}\right], \boldsymbol{I}=\left[\begin{array}{l}
I_{\mathrm{d}} \\
I_{\mathrm{q}}
\end{array}\right], \boldsymbol{A}_{\mathrm{P}}=\left[\begin{array}{cccc}
-1 /\left(\tau_{\mathrm{m}}+3 T_{\mathrm{d}}\right) & 0 & 0 & 0 \\
1 / T_{\mathrm{d} 0}^{\prime} & -1 / T_{\mathrm{d} 0}^{\prime} & 0 & 0 \\
c / T_{\mathrm{d} 0}^{\prime} & 1 / T_{\mathrm{d} 0}^{\prime \prime}-c / T_{\mathrm{d} 0}^{\prime} & -1 / T_{\mathrm{d} 0}^{\prime \prime} & 0 \\
0 & 0 & 0 & -1 / T_{\mathrm{q} 0}^{\prime \prime}
\end{array}\right], \boldsymbol{C}_{\mathrm{P}}=\left[\begin{array}{l}
0 \\
1 \\
0 \\
0
\end{array}\right]^{\mathrm{T}}, \\
& \boldsymbol{B}_{\mathrm{P} 1}=\left[\begin{array}{cc}
0 & 0 \\
\left.\left(X_{\mathrm{d}}^{\prime}-X_{\mathrm{d}}\right) / T_{\mathrm{d} 0}^{\prime \prime}-X_{\mathrm{d}}^{\prime}\right) / T_{\mathrm{d} 0}^{\prime \prime}+\left(c X_{\mathrm{d}}^{\prime}-X_{\mathrm{d}}\right) / T_{\mathrm{d} 0}^{\prime} & 0 \\
0 & \left(X_{\mathrm{q}}-X_{\mathrm{q}}^{\prime \prime}\right) / T_{\mathrm{q} 0}^{\prime \prime}
\end{array}\right], \boldsymbol{B}_{\mathrm{P} 2}=\left[\begin{array}{c}
\frac{K_{\mathrm{PWM}}\left(X_{\mathrm{d}}-X_{1}\right)}{R_{\mathrm{f}}\left(\tau_{\mathrm{m}}+3 T_{\mathrm{s}}\right)} \\
0 \\
0 \\
0
\end{array}\right] .
\end{aligned}
$$

Finally, the differential Equations shown from (4) to (11) can be discreted to incremental Equations by using the finite difference method.

\subsubsection{Control Objectives}

When operated under different wind speed condition during LVRT, the control objectives of the coordination controller can be summarized in three aspects:

1) The first objective is to minimize the variation of the output power, which can be described as

$$
\begin{aligned}
& J_{1}=\min f_{1}(\beta, \theta)=\min \left(\frac{\omega_{\mathrm{G}}-\omega_{\mathrm{ref}}}{\omega_{\mathrm{ref}}}\right) \\
& \text { s.t. }\left\{\begin{array}{l}
0 \leq \beta \leq \beta_{\max } \\
0 \leq \theta \leq \theta_{\max } \\
0 \leq \omega_{\mathrm{G}} \leq \omega_{\mathrm{G} \max }
\end{array}\right.
\end{aligned}
$$

where the objective function can be derived from (3) to (6).

2) The second objective is to keep the rotor speed of EESG in a set range. The control objectives function can be donated as

$$
J_{3}=\min f_{2}(\beta, \theta)=\min \left(\frac{P_{\mathrm{G}}-P_{\mathrm{ref}}}{P_{\mathrm{ref}}}\right)
$$


s.t. $\left\{\begin{array}{l}0 \leq P_{\mathrm{G}} \leq P_{\max } \\ 0 \leq \beta \leq \beta_{\text {max }} \\ 0 \leq \theta \leq \theta_{\text {max }}\end{array}\right.$

where $P_{\text {ref }}$ represents the reference output power of generator, and the output active power of EESG is

$$
P_{\mathrm{e}}=\frac{U E_{\mathrm{q}}}{X_{\mathrm{d}}} \sin \delta+\left(\frac{1}{X_{\mathrm{q}}}-\frac{1}{X_{\mathrm{d}}}\right) \frac{U^{2}}{2} \sin 2 \delta
$$

3) The third objective is to keep the stator voltage of EESG in a normal level, donates the stator voltage is $V_{\mathrm{s}}$, the objective function is

$$
\begin{aligned}
& J_{2}=\min \Delta V_{t}\left(\delta, E_{q}^{\prime}\right) \\
& \text { s.t. } 0 \leq V_{t} \leq 1.2 V_{R}
\end{aligned}
$$

where $\Delta V_{\mathrm{t}}=a \Delta \delta+b \Delta E_{q}^{\prime}$ is the stator voltage Equation of EESG which can describe the stator voltage variation along with power angle $\delta$ and $E_{\mathrm{q}}^{\prime}$.

\section{Simulation and Results}

During voltage sagging, the LVRT controller would take in charge of the WT until PCC voltage recovered. Figure 7 gives the flow chart for LVRT control of FSCWT, which was mainly depend on the LVRT requirements for WT of grid codes shown in Figure 3.

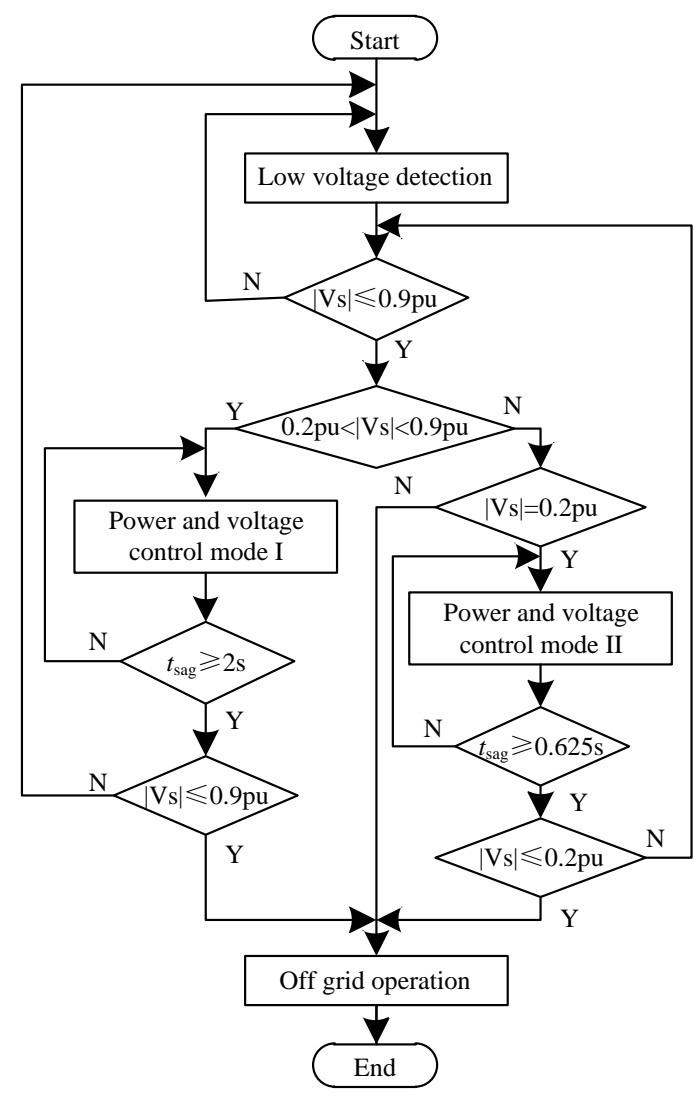

Figure 7. Flow Chart for LVRT Control of FSCWT

To a symmetrical three phase fault, there is no negative sequence voltage existed. Thus, the d-q transformation (2s/2r) method [20] can be utilized for voltage sag detection. 


$$
\begin{aligned}
& {\left[u_{\mathrm{d}}, u_{\mathrm{q}}\right]^{\mathrm{T}}=\mathrm{C}\left[u_{\mathrm{a}}, u_{\mathrm{b}}, u_{\mathrm{c}}\right]^{\mathrm{T}}} \\
& \text { with } \\
& C=\sqrt{\frac{2}{3}}\left[\begin{array}{lll}
\sin \omega t & \sin (\omega t-2 \pi / 3) & \sin (\omega t+2 \pi / 3) \\
-\cos \omega t & -\cos (\omega t-2 \pi / 3) & -\cos (\omega t+2 \pi / 3)
\end{array}\right]
\end{aligned}
$$

Thus, the sag time and the sag amplitude can be detected by the voltage variation in d-axis. Figure 8 shows the low voltage detection results with $60 \%$ and $40 \%$ sag, respectively. As can be seen from the figure, the waveform of voltage $u_{\mathrm{d}}$ can detect the voltage sags without any time delay when it dropped at $0.032 \mathrm{~s}$ and return in a normal value at $0.082 \mathrm{~s}$.

To the asymmetrical fault, like one phase or two phase short circuit of grid side, an improved d-p transformation method without time delay was employed, where the voltage of the fault phase was implemented with derivative, the derived cosine voltage is utilized for structuring a symmetrical three phase voltage Equation. Then, the d-q transformation can be utilized to detect the voltage sag.
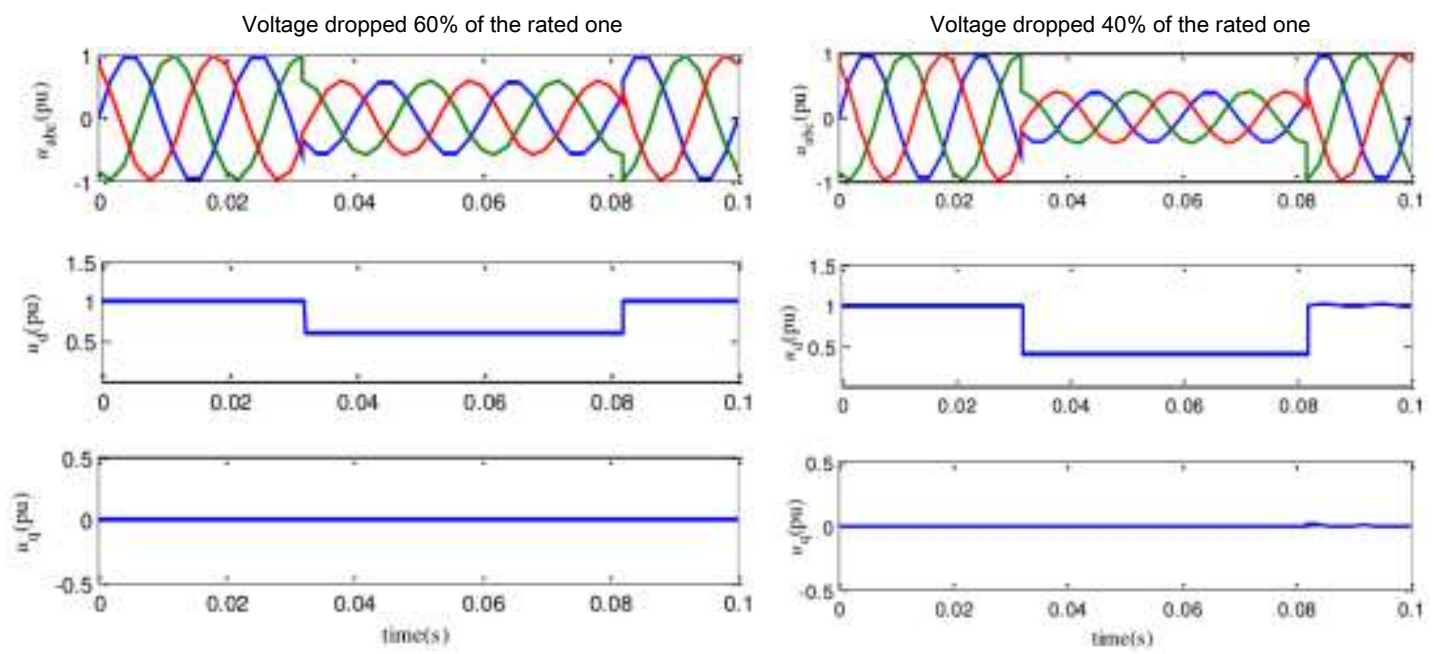

Figure 8. Low Voltage Detection Results with Different Sags

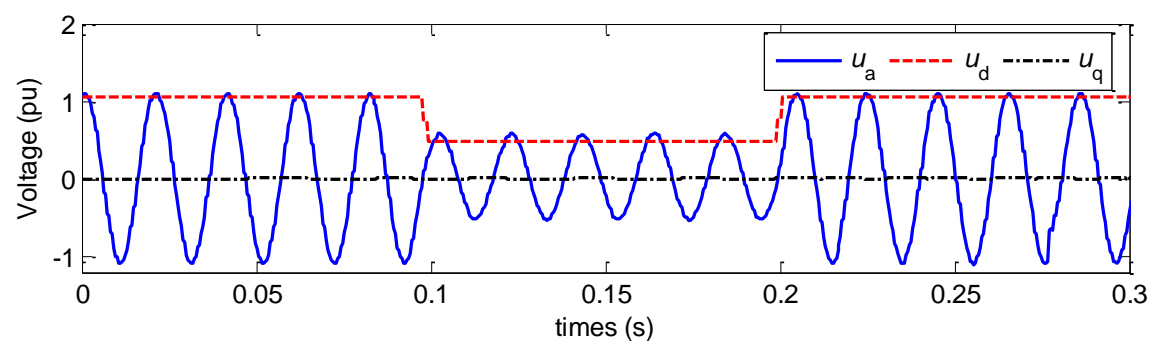

Figure 9. Low Voltage Detection Results in Phase A

Assuming voltage sag occurred in phase A, the deviation of $u_{\mathrm{a}}$ is

$$
u_{a}^{\prime}=U \omega \cos \omega t
$$

according to triangular transformation

$$
\left\{\begin{array}{l}
u_{b}=-\frac{1}{2} u_{a}-\frac{3}{2 \omega} u_{a}^{\prime}(t) \\
u_{c}=-\frac{1}{2} u_{a}+\frac{3}{2 \omega} u_{a}^{\prime}(t)
\end{array}\right.
$$

The d-q transformation result is 


$$
\left\{\begin{array}{l}
u_{d}=\sqrt{3} U \cos \alpha \\
u_{q}=\sqrt{3} U \sin \alpha
\end{array}\right.
$$

Figure 9 shows the low voltage detection results obtained in phase A, it obviously that there is no time delay of $u_{\mathrm{d}}$ when the voltage dropped at $0.1 \mathrm{~s}$ and returned at $0.2 \mathrm{~s}$. Therefore, both the symmetrical and asymmetrical methods are used for low voltage detection in this paper as the first step for the LVRT controller.

For the purpose of improving the control performance, and reducing the calculation tasks when solving the nonlinear optimal problem proposed in this paper, the step-like control is utilized. Assuming the timely control increment is

$$
\Delta u(k)=u(k)-u(k-1)
$$

with a step coefficient $\gamma \geq 0$. The future control increment can be expressed as

$\Delta u(k+j)=\gamma \Delta u(k+j-1)=\gamma^{j} \Delta u(k) \quad 1 \leq j \leq p-1$

where $p$ is the prediction length. Thus, the future control sequence $u(k)$, $u(k+1), \ldots, u(k+p-1)$ can be described using $u(k-1)$ and $\Delta u(k)$. The dimensions is reduced from $i \cdot p$ to $i$, which would be only related to the input dimension. In this paper, the prediction length $p$ is set to 20 with a step coefficient $\gamma=0.8$.

Ranking the control objectives in the priorities: $J_{3}>J_{2}>J_{1}$. The corresponding response curves are shown in Figure 10. Defining both the limit values in (12) and (13) with $\beta_{\max }=90^{\circ}, \theta_{\max }=90^{\circ}, \omega_{\mathrm{G} \max }=1.2 \omega_{\mathrm{GR}}$ and all set values are equal to " 1 " in per unit. Figure 10 shows the simulation results with the proposed method used when the voltage of PCC sagged to $85 \%$ and $20 \%$, respectively, with a three phase short happen in grid side.
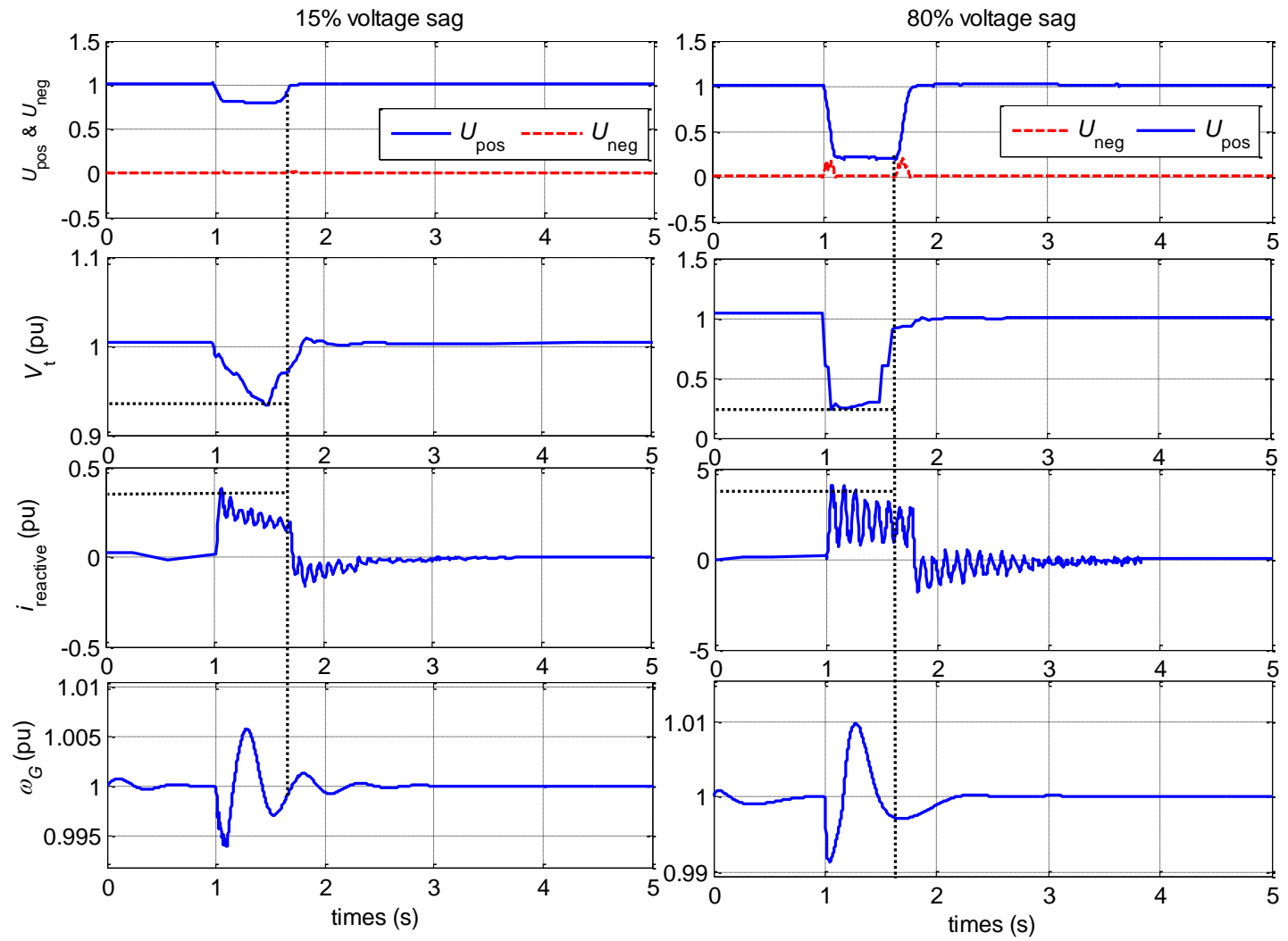

Figure 10. LVRT Characteristics with Different Voltage Sag

It can be seen from the figure that when a three phase fault happen in the grid side at $1 \mathrm{~s}$, it can be timely detected by $U_{\text {pos }}$ without any delay. When the fault is cleared at $1.625 \mathrm{~s}$, the terminal voltage recovers within a very short time. In the case when the voltage 
sagged to $20 \%$ of the rated value, which only takes $180 \mathrm{~ms}$ and fully meets with the requirements of LVRT capabilities for WTs. The maximal rotor speed variation during three phase short circuit was only $1 \%$ with $80 \%$ voltage sag while $5.2 \%$ for a $15 \%$ voltage sag. During the fault, the reactive current was injected rapidly for supporting the voltage, with a maximal $3.8 \mathrm{pu}$ in the worst case.

To exemplify the superiority of the proposed method, a comparison of the proposed method and $H_{\infty}$ strategy, proposed by the authors for the same purpose [21], which is shown in Figure 11.

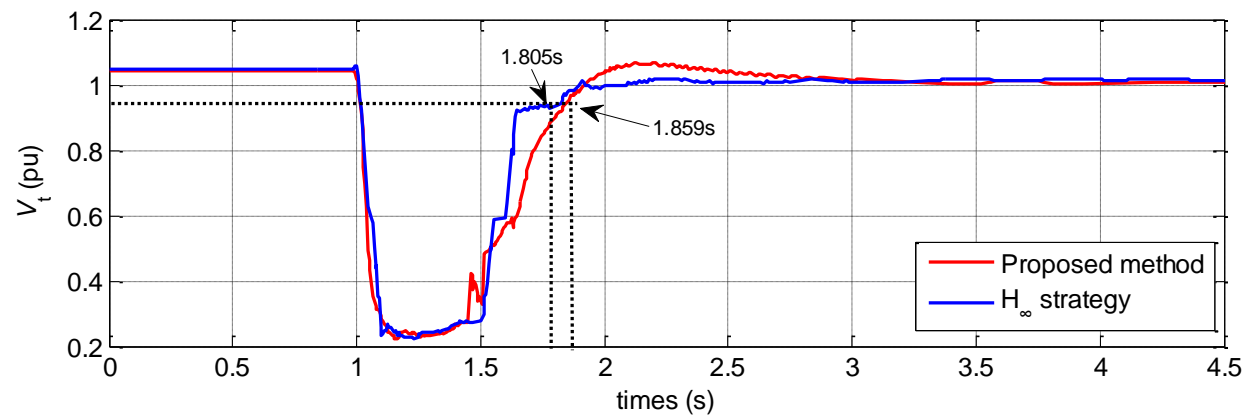

Figure 11. LVRT Characteristics Comparison with $\mathrm{H}_{\infty}$ Strategy

As shown in Figure 11, when the same three faults happened at 1s and cleared at $1.625 \mathrm{~s}$, the voltage under the control of proposed method can quickly recover to $90 \%$ of the voltage to its normal operating standard at $1.805 \mathrm{~s}$, which is $54 \mathrm{~ms}$ higher than that voltage under $\mathrm{H}_{\infty}$ control. All this two approaches can meet the LVRT requirements for WTs. Figure 12 shows the active power variation during normal operation without any faults occurred. It is obvious that the output power is smoother when the priority of the three objective functions is $J_{1}>J_{2}>J_{3}$ rather than $J_{2}>J_{1}>J_{3}$.

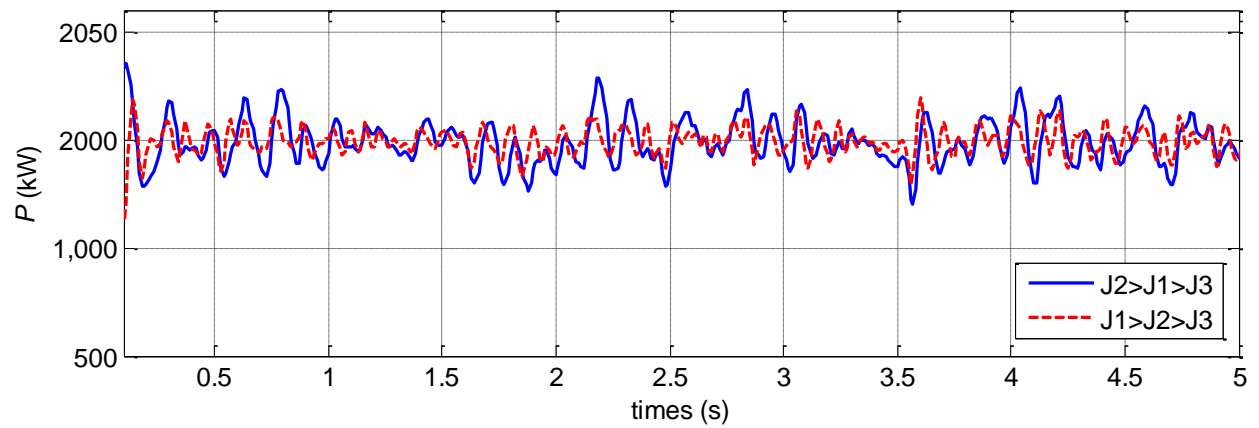

Figure 12. Output Power with Different Objective Priorities

As we can see from the figure, during the operation of the FSCWT under different conditions or different operation zones (see in Figure 2), different objectives require different priorities to fulfill their set targets. The proposed method adopts the nonlinear multi-object predictive strategy to cope with such demands, which is proved with good performance.

\section{Conclusions}

In this paper, the multi-object predictive strategy has been applied for coordinating control of pitch control, excitation control and WinDrive control for improving the LVRT capability of FSCWT. With interpreting the operation principle as well as the LVRT requirements for FSCWT, the proposed method was verified by simulations conducted in 
MATLAB environment. Experimental results show that when the WT operating in different zones, better performances can be obtained by adjusting the priority of the objectives. Comparison study of LVRT capability with proposed method as well as an addition $\mathrm{H}_{\infty}$ strategy utilized show that the proposed method is of stronger robustness.

\section{Acknowledgements}

[1] This paper was funded by the 863 Project of China (2012AA052903); Nature Science Foundation (Collaborative) of Gansu (213244); and Youth Science Foundation of Lanzhou Jiaotong University (2013040).

\section{References}

[1] Erlich and U. Bachmann, "Grid code requirements concerning connection and operation of wind turbines in Germany", Proceedings of IEEE Power Engineering Society General Meeting, Denver, USA, (2005).

[2] H. M. EL-Helw and S. B. Tennakoon, "Evaluation of the suitability of a fixed speed wind turbine for large scale wind farms considering the new UK grid code", Renewable Energy, vol. 33, no. 1, (2008), pp. 1-12.

[3] L.A. Trujillo Guajardoa, A. Conde Enríqueza and Z. Leonowiczb, "Error compensation in distance relays caused by wind power plants in the power grid", Electric Power Systems Research, vol. 106, (2014), pp. 109-119.

[4] Ahmidi, X. Guillaud, Y. Besanger and R. Blanc, "A multilevel approach for optimal participating of wind farms at reactive power balancing in transmission power system," IEEE System Journal, vol. 6, no. 2, (2012), pp. 260-269.

[5] M. El-Shimy, "Modeling and analysis of reactive power in grid-connected onshore and offshore DFIGbased wind farms", Wind Energy, vol. 17, no. 2, (2014), pp. 279-295.

[6] T. Ackermann, "Wind Power in Power Systems", John Wiley \& Sons, (2005).

[7] Murukeshan and R. Rajasekaran, "Power system stability enhancement and improvement of LVRT capability of a DFIG based wind power system by using SMES and SFCL", International Journal of Electric \& Computer Engineering, vol. 3, no. 5, (2013), pp. 618-628.

[8] J. Ma, X. Lan, X. Ding and Z. Wang, "Transient characteristics of symmetrical short circuit fault in double fed induction generators considering grid-side converter control and phase-angle jump of DFIG's terminal voltage", Power System Technology, vol. 38, no. 7, (2014), pp. 1891-1897.

[9] Besteck, "WinDrive-variable speed wind turbines without converter with synchronous generator", Technique report, (2009).

[10] "Modeling and verification method of wind turbines for low voltage ride through", NB/T 31051-2014, National Energy Administration, Xinhua press, (2014).

[11] S. Bharat and S. N. Singh, "Wind power interconnection into the power system: a review of grid code requirements", The Electricity Journal, vol. 22, no.5, (2009), pp. 54-63.

[12] Utilities Research Institute of Danish Electric, KR111-E, "Connection of wind turbines to low and medium voltage networks", (1998), pp. 3-8.

[13] M. Ivan and A. Itzel, "Grid codes comparison”, Göteborg: Chalmers University of Technology, (2006), pp. 31-45.

[14] Z. Tao, W. Gang and H. Defeng, "Nonlinear model predictive control based on lexicographic multiobjective genetic algorithm", Proceedings of International Conference on Intelligent \& Advanced System, Kuala Lumpur, Malaysia, (2007).

[15] Z. Tao, W. Guang and L. Guanghong, "Stratified multi-objective nonlinear model predictive control", Journal of Jiangnan University (Natural Science Edition), vol. 9, no. 4, (2010), pp. 440-443.

[16] N. Peter, E. Thommy and J. Inge, "Modeling and control of variable-speed wind turbine drive-system dynamics", IEEE Transactions on Control Systems, vol. 15, no. 4, (1995), pp. 28-38.

[17] J. G. Slootweg, S.W.H. De Haan and H. Polinder, "General model for representing variable speed wind turbines in power system dynamics simulations", IEEE Transactions on Power Systems, vol. 18, no. 1, (2003), pp. 144-151.

[18] W. Ma, X. Liu and C. Liu, "Hydrodynamic speed adjusting system and its control of wind turbine", Journal of Jilin University (Nacture Science Edition), vol. 43, no. 5, (2013), pp. 1276-1283.

[19] E. Mouni, and S. Tanai, "Synchronous generator output voltage control via a generalized predictive R S T controller", IEEE International Symposium on Industrial Electronics, (2008), pp. 718-723.

[20] G. Zhao, B. Liu, X. Xiao and Y. Xu, "Application of improved d-q transform without time delay in dynamic voltage disturbance identification”, Power System Technology, vol. 28, no. 7, (2004), 53-57.

[21] H. Dong, S. Li, L. Cao and H. Li, "Voltage and Reactive Power Control of Front-end Speed Controlled Wind Turbine via $\mathrm{H}_{\infty}$ Strategy", TELKMONIKA Indonesia Journal of Electric and Electronic Engineering, vol. 11, no. 8, (2013), pp. 4190-4199. 


\section{Authors}

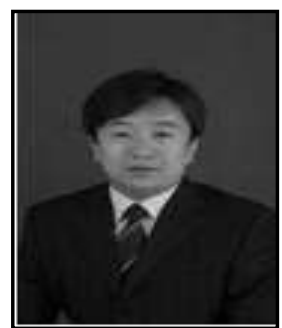

Hongwei Li, he was born in Gansu of China in 1981, received his bachelor's and master's degree in Lanzhou Jiaotong University in 2003 and 2009 respectively, both majoring in Traffic Information Engineering Control. From 2003 to 2012, he was a research engineer with the school of Automation and Electrical Engineering in Lanzhou Jiaotong University, where he was engaged in the research of control of wind generation systems. Since August 2012, he has been studying for his PhD. His current research interests are in the areas of coordination control of subsystems of wind turbine. 